\title{
Flux-correlation approach to characterizing reaction pathways in quantum systems: A study of condensed-phase proton-coupled electron transfer
}

\author{
Nandini Ananth ${ }^{1}$ and Thomas F. Miller, III ${ }^{1}$ \\ Division of Chemistry and Chemical Engineering, California Institute of Technology, Pasadena, California 91125, \\ $U S A$
}

We introduce a simple method for characterizing reactive pathways in quantum systems. Flux autocorrelation and cross-correlation functions are employed to develop a quantitative measure of dynamical coupling in quantum transition events, such as reactive tunneling and resonant energy transfer. We utilize the method to study condensed-phase proton-coupled electron transfer (PCET) reactions and to determine the relative importance of competing concerted and sequential reaction pathways. Results presented here include numerically exact quantum dynamics simulations for model condensed-phase PCET reactions. This work demonstrates the applicability of the new method for the analysis of both approximate and exact quantum dynamics simulations.

\section{INTRODUCTION}

Detailed mechanistic understanding of condensedphase chemical dynamics is essential for the design of next-generation molecular catalysts and photosystems. The role of numerical simulations in this effort is particularly important for systems that exhibit multiple, competing reactive pathways. The characterization of reactive pathways in classical systems has been greatly advanced by the development of methods for rare event sampling ${ }^{1}$. However, the corresponding tools for quantum systems ${ }^{7-11}$ are less developed, despite significant demand for understanding reactive charge and energy transfer pathways in complex quantum systems.

In this paper, we introduce a method for characterizing reactive pathways and quantifying dynamical correlations in general quantum systems using real-time flux auto-correlation (FAC) and flux cross-correlation (FCC) functions. We employ the new approach to investigate the reaction dynamics of condensed-phase protoncoupled electron transfer (PCET), an important prototype for quantum systems that exhibit multiple reaction pathways 12 . Numerical results demonstrate the practicality and utility of the new method for the mechanistic analysis of coupled quantum dynamical processes.

\section{THEORY}

We consider a set of states $\{|n\rangle\}$ that span the full Hilbert space of a closed quantum system, and we consider the partitioning of this set of states into $N$ subsets $\left\{\Omega_{j}\right\}$, such that each state belongs to one and only one subset. The criteria for this partitioning are flexible; for instance, cut-offs based on energy expectation values can be used to generate subsets of states with similar energies, and conditions based on position expectation values can be used to generate subsets of states with similar configurations. The dividing surface corresponding to subset
$\Omega_{j}$ is defined in terms of the projection operator

$$
\mathcal{P}_{j}=\sum_{i=1}^{\text {all states }}\left|n_{i}\right\rangle\left\langle n_{i}\right| \epsilon_{i j},
$$

where $j=1, \ldots N$, and

$$
\epsilon_{i j}= \begin{cases}1, & \left|n_{i}\right\rangle \in \Omega_{j} \\ 0, & \left|n_{i}\right\rangle \notin \Omega_{j}\end{cases}
$$

The net flux associated with transitions from subset $\Omega_{j}$ to the remainder of the Hilbert space is given by

$$
F_{j}=\frac{i}{\hbar}\left[H, \mathcal{P}_{j}\right]
$$

where $H$ is the Hamiltonian for the full system. Dynamical correlations between these transitions can be obtained from thermal, real-time FAC and FCC functions ${ }^{7 / 19} \mid \sqrt{22}$,

$$
C_{m j}(t)=\operatorname{Tr}\left[e^{-\beta H} F_{m} e^{i H t} F_{j} e^{-i H t}\right],
$$

where $\beta$ is the reciprocal temperature, and the FAC function corresponds to the case $m=j$. Physically, Eq. 4 4 describes the correlation between transitions into/out-of subset $\Omega_{m}$ with transitions into/out-of subset $\Omega_{j}$.

Using that the net flux for a closed quantum system is zero, i.e. $\sum_{j=1}^{N} F_{j}=0$, the FAC function can be expressed as a linear combination of FCC functions; specifically,

$$
C_{j j}(t)=-\sum_{m=1}^{N} C_{m j}(t)
$$

where the summation excludes the $m=j$ term. Introducing the zeroth moments of the real part of these correlation functions,

$$
k_{m j}=\int_{0}^{\infty} d t \operatorname{Re}\left[C_{m j}(t)\right]
$$


Eq. (5) then yields

$$
k_{j j}=-\sum_{m=1}^{N} k_{m j} .
$$

Within the assumption that $\mathcal{P}_{j}$ divides the system into two basins of stability, Eq. (7) provides a decomposition of the corresponding reaction rate. We thus introduce a measure of the degree to which dynamical correlations between transitions associated with subsets $\Omega_{j}$ and $\Omega_{m}$ contribute to the overall reaction rate,

$$
\kappa_{m j}=-\frac{k_{m j}}{k_{j j}} .
$$

More generally, regardless of metastability of subsets, the dynamical correlation factor (DCF) defined in Eq. (8) provides a transferable measure of the relative contribution from subset $\Omega_{m}$ to the transient dynamics associated with entering/leaving subset $\Omega_{j}$. Non-zero values for the DCF indicate correlated transitions between the subsets and provide a basis for identifying important dynamical pathways and reaction mechanisms. We note that the DCF can be calculated using either exact or approximate quantum dynamical methods $\sqrt{23}$, and flexibility in the definition of subset partitions enables the detailed characterization of complex systems. Furthermore, to characterize processes that are far from thermal equilibrium, DCFs can be similarly constructed in terms of flux correlation functions with non-Boltzmann initial distributions.

\section{PCET MODEL SYSTEMS}

To demonstrate this flux-correlation approach for the analysis of systems with competing dynamical pathways, we consider a series of condensed-phase PCET reactions. The reactions are described using a system-bath Hamiltonian,

$$
\begin{aligned}
H= & \frac{p_{s}^{2}}{2 m_{s}}+\frac{p_{x}^{2}}{2 m_{x}}+V_{\mathrm{p}}(x)+V_{\mathrm{ps}}(x, s)+V_{e}(x, s)+ \\
& +\sum_{j} \frac{P_{j}^{2}}{2 M_{j}}+\frac{1}{2} M_{j} \omega_{j}^{2}\left(Q_{j}-\frac{c_{j} s}{M_{j} \omega_{j}^{2}}\right)^{2},
\end{aligned}
$$

where the coordinates $x, s$ and $Q_{j}$ correspond to the proton, solvent polarization, and bath modes with masses of $m_{x}, m_{s}$, and $M_{j}$, respectively. Potential energy surfaces associated with the donor and acceptor electronic states are described in the diabatic representation,

$$
V_{e}(x, s)=\left(\begin{array}{cc}
V_{11}(s)+V_{\mathrm{ep}}(x) & V_{12}(s) \\
V_{12}(s) & V_{22}(s)-V_{\mathrm{ep}}(x)
\end{array}\right),
$$

where $V_{i i}(s)=\frac{1}{2} m_{s} \omega_{s}^{2}\left(s \quad-\quad s_{i}\right)^{2} \quad$ with $i=1,2$, the electron-proton coupling is given by $V_{\text {ep }}(x)=\mu_{2} \tanh (\phi x)$, and $V_{12}(s)$ is the diabatic coupling.
The term $V_{\mathrm{p}}(x)$ in Eq. (9) describes a double-well potential for the proton coordinate,

$$
V_{\mathrm{p}}(x)=-\frac{m_{x} \omega_{x}^{2}}{2} x^{2}+\frac{m_{x}^{2} \omega_{x}^{4}}{16 V_{0}} x^{4}-\lambda x^{3},
$$

where $\omega_{x}$ is the proton vibrational frequency, and $V_{0}$ is the intrinsic proton transfer barrier height. The proton solvent interaction is described using $V_{\mathrm{ps}}(x, s)=-\mu_{1} s \tanh (\phi x)$.

The dissipative bath in Eq. (9) exhibits an Ohmic spectral density,

$$
J(\omega)=\eta \omega e^{-\omega / \omega_{c}},
$$

where $\omega_{c}$ is the cut-off frequency, and $\eta$ is the dimensionless coupling between solvent and bath modes 24 . The spectral density is discretized using $f$ oscillators with frequencies 25

$$
\omega_{j}=-\omega_{c} \log \left(\frac{j-0.5}{f}\right)
$$

and coupling constants

$$
c_{j}=\omega_{j}\left(\frac{2 \eta M_{j} \omega_{c}}{f \pi}\right),
$$

where $j=1, \ldots f$.

PCET reactions proceed via concerted or sequential mechanisms, depending on the chronology of the electron transfer (ET) and proton transfer (PT) events. The concerted mechanism involves dynamically correlated transfer of both the electron and proton, whereas the sequential mechanism involves dynamically uncorrelated ET and PT steps. We consider three models for PCET in the current study, each of which corresponds to a different set of parameters for the Hamiltonian in Eq. (9). Models I and II are adapted from an earlier study of unidirectional PCET in non-dissipative systems and assume that the solvent responds to the transferring electron and proton as if they are like-charged particles 26 . This unphysical picture of solvent polarization is corrected in model III, in which the solvent response to the transferring electron is counteracted by the solvent response to the transferring proton. The parameters for all three models are provided in Table 1 .

\section{CALCULATION DETAILS}

For each PCET model system, FAC and FCC functions are calculated using the quasi-adiabatic path-integral (QUAPI) method ${ }^{27}$ 31. QUAPI is a numerically exact quantum dynamics method that employs a real-time path integral formulation. It has previously been used to study single-particle transfer reactions, such as ET or PT 30131 ; here, we extend the approach to describe the coupled transfer of both an electron and a proton. Details of the QUAPI implementation are provided in Appendix I. 
TABLE I. Parameters for the model PCET systems.

\begin{tabular}{cccc}
\hline Parameter $^{\mathrm{a}}$ & Model I & Model II & Model III \\
\hline$m_{x}$ & 1836.1 & 1836.1 & 1836.1 \\
$\omega_{x}$ & 0.0104 & 0.0104 & 0.0104 \\
$V_{0}$ & 0.012 & 0.014 & 0.012 \\
$m_{s}$ & 22000 & 22000 & 22000 \\
$\omega_{s} \times 10^{4}$ & 3.72 & 4.00 & 3.72 \\
$s_{1}$ & -2.13 & -2.16 & -2.13 \\
$s_{2}$ & 2.13 & 2.16 & 2.13 \\
$V_{12}$ & 0.0245 & 0.0124 & 0.0245 \\
$\mu_{1}$ & 0.0011 & 0.017 & -0.0011 \\
$\mu_{2} \times 10^{3}$ & 5.84 & 0.71 & 5.84 \\
$\phi$ & 8.0 & 8.0 & 8.0 \\
$\lambda$ & 0.0 & 0.012 & 0.0 \\
$f$ & 12 & 12 & 12 \\
$M_{j}$ & $m_{s}$ & $m_{s}$ & $m_{s}$ \\
$\eta$ & $m_{s} \omega_{s}$ & $m_{s} \omega_{s}$ & $m_{s} \omega_{s}$ \\
$T / \mathrm{K}$ & 300 & 750 & 300 \\
\hline \hline
\end{tabular}

a All values in atomic units, unless otherwise specified.

We partition the full set of quantum states for the PCET Hamiltonian (Eq. (9) ) into $N=3$ subsets that are defined in terms of the proton position and the electronic diabatic state. The subset associated with the PCET reactant states, $\Omega_{\mathrm{r}}$, is defined in terms of the projection operator

$$
\mathcal{P}_{\mathrm{r}}=|1\rangle\langle 1| h(-x) .
$$

Similarly, $\Omega_{\mathrm{p}}$ includes the PCET product states and is defined using

$$
\mathcal{P}_{\mathrm{p}}=|2\rangle\langle 2| h(x),
$$

and $\Omega_{\mathrm{i}}$ includes intermediate states associated with single-particle transfer and is defined using

$$
\mathcal{P}_{\mathrm{i}}=|1\rangle\langle 1|h(x)+| 2\rangle\langle 2| h(-x) .
$$

In these equations, $|1\rangle$ and $|2\rangle$ indicate the donor and acceptor electronic diabatic states, and $h(x)$ is the heaviside function

$$
h(x)= \begin{cases}1, & x>0 \\ 0, & x<0\end{cases}
$$

Using these subset definitions, the corresponding FAC and FCC functions are calculated using Eq. (4). Finally, Eq. (8) is used to calculate $\kappa_{\text {ip }}$ and $\kappa_{\text {rp }}$, which quantify dynamical correlations of the product subset with the intermediate and reactant subsets, respectively. For the present case in which the states are simply partitioned into reactant, product, and intermediate subsets, we can relate the calculated DCF to competing reaction mechanisms. Specifically, by reporting on whether the reaction dynamics proceeds via the intermediate subset, or whether it proceeds via direct transfer from the reactant to product subsets, $\kappa_{\mathrm{ip}}$ and $\kappa_{\mathrm{rp}}$ respectively indicate the degree to which the sequential or concerted PCET mechanism is dominant.

\section{RESULTS}

Fig. 1 presents the calculated FAC and FCC functions for model I, with $C_{\mathrm{pp}}(t), C_{\mathrm{rp}}(t)$, and $C_{\mathrm{ip}}(t)$ plotted in blue, red, and green, respectively. The FAC function exhibits initial decay on the 500 a.u. timescale, followed by modest recrossing. Cross-correlations in the subset dynamics are most pronounced for the reactant and product subsets, with $C_{\mathrm{rp}}(t)$ closely mirroring the features of the product FAC function. In contrast, only a small degree of cross-correlation is found for the dynamics associated with the intermediate and product subsets. All dynamical correlations among the subsets are found to vanish by approximately 4000 a.u. in time.

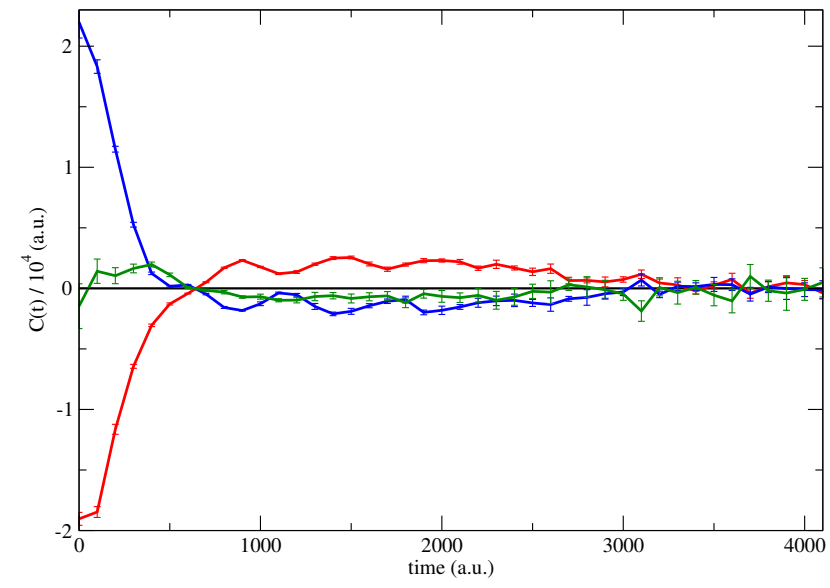

FIG. 1. Numerically exact, symmetrized flux-correlation functions for model I, with $C_{\mathrm{pp}}(t)$ in blue, $C_{\mathrm{rp}}(t)$ in red, and $C_{\mathrm{ip}}(t)$ in green.

Integration of these correlation functions yields the DCF for model I, which are reported in Table II. The larger magnitude of $\kappa_{\mathrm{rp}}$ indicates that the PCET reaction in model I primarily proceeds via the concerted mechanism, although a substantial contribution from the sequential pathway is also found. We note that earlier simulations for a non-dissipative version of this PCET model also concluded the importance of the concerted mechanism $26 \mid 32$.

TABLE II. Reaction mechanisms for PCET in models I-III.

\begin{tabular}{ccccc}
\hline \hline Pathway & DCF & Model I & Model II & Model III \\
\hline Concerted & $\kappa_{\mathrm{rp}}$ & $0.62(6)$ & $0.010(7)$ & $0.93(7)$ \\
Sequential & $\kappa_{\mathrm{ip}}$ & $0.38(5)$ & $0.99(5)$ & $0.07(3)$ \\
\hline \hline
\end{tabular}

Fig. 2 presents the $C_{\mathrm{pp}}(t), C_{\mathrm{rp}}(t)$, and $C_{\mathrm{ip}}(t)$ correlation functions for model II at short times, which reveal significant differences in comparison to Fig. 1. The FAC function for model II exhibits pronounced oscillations on the timescale of proton vibrations, but more striking differences are seen in the cross-correlation functions. In Fig. 2. far greater contributions are seen from the $C_{\text {ip }}$ than $C_{\text {rp }}$, indicating that flux into the product state is 
dynamically coupled with the single-particle transfer intermediates, rather than with the reactant subset. This point is further illustrated in Table II] which reveals $\kappa_{\text {ip }}$ to approach unity while $\kappa_{\mathrm{rp}}$ nearly vanishes. These results clearly indicate that model II exhibits a sequential PCET reaction mechanism, in agreement with studies of a related, non-dissipative system $26[32$.

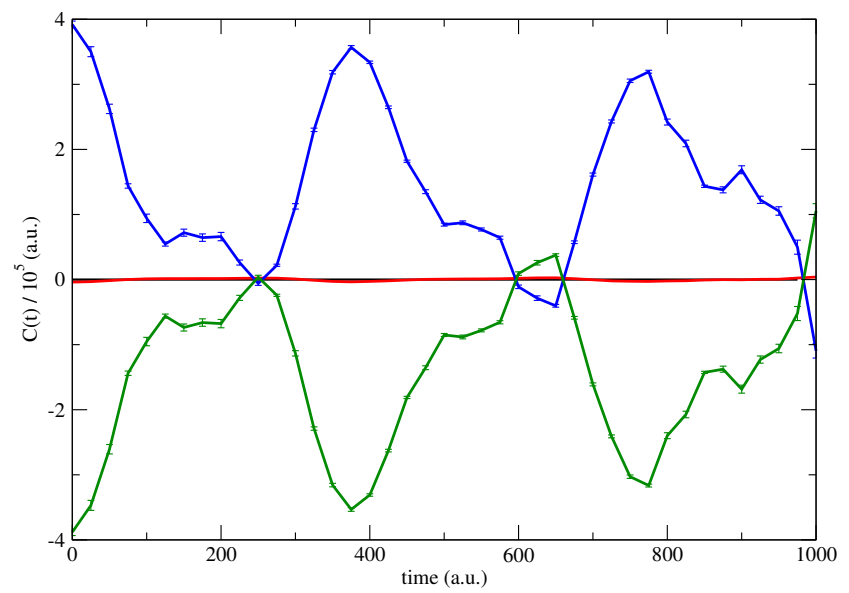

FIG. 2. Numerically exact, symmetrized flux-correlation functions for model II, with $C_{\mathrm{pp}}(t)$ in blue, $C_{\mathrm{rp}}(t)$ in red, and $C_{\mathrm{ip}}(t)$ in green.

Finally, Fig. 3 presents the flux-correlation functions for model III, which differs from model I only in terms of the solvent polarization response to the proton coordinate (Table I). The FAC function for model III exhibits a slower timescale for initial decay than for model I, as well as a more pronounced degree of dynamical recrossing. Both FCC functions reflect the time dependence of the FAC function, although the magnitude of $C_{\mathrm{rp}}(t)$ is greater than that of $C_{\mathrm{ip}}$ at all times. Interestingly, Table II shows that $\kappa_{\text {rp }}$ approaches unity, whereas $\kappa_{\text {ip }}$ nearly vanishes, an indication that the PCET reaction in model III is more dominated by the concerted mechanism than the corresponding concerted reaction in model I. In model III, the solvent response to the net-neutral PCET charge-transfer reaction yields reactant and product states with less solvent polarization than in model I; the energetic favorability of minimizing solvent reorganization throughout the reaction thus creates a driving force for co-localizing the charge distributions of the transferring electron and proton, which leads to the more strongly concerted PCET mechanism observed for model III.

\section{CONCLUSIONS}

We introduce a method in which flux-correlation functions are used to characterize dynamical correlations and reaction pathways in quantum systems. Numerical results demonstrate the utility of the method for the analysis of exact quantum dynamic simulations of condensed-

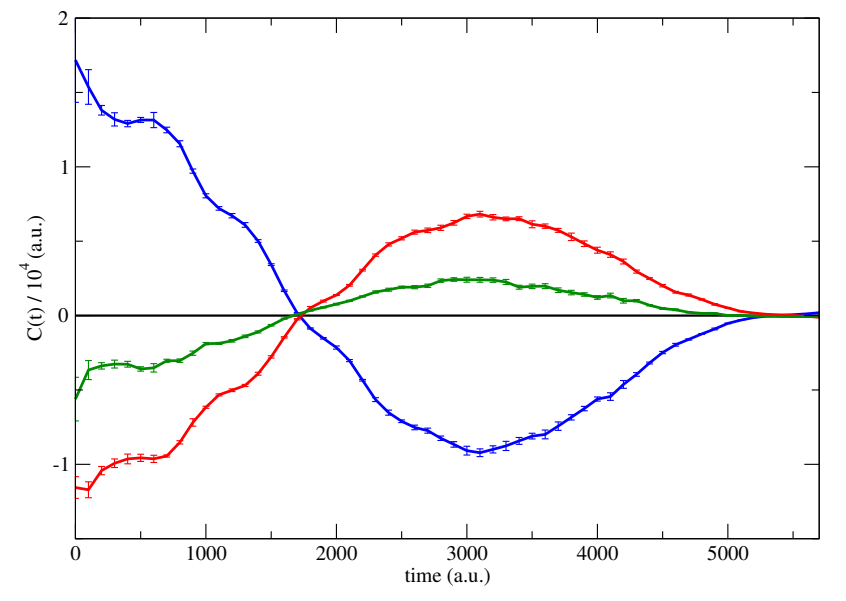

FIG. 3. Numerically exact, symmetrized flux-correlation functions for model III, with $C_{\mathrm{pp}}(t)$ in blue, $C_{\mathrm{rp}}(t)$ in red, and $C_{\mathrm{ip}}(t)$ in green.

phase PCET. In particular, we quantify the relative importance of the sequential and concerted PCET reaction mechanisms for two previously studied model systems, and in a third system, we demonstrate that introduction of a refined description for the solvent-proton coupling leads to greater dominance of the concerted PCET mechanism. It is expected that this approach will prove useful in the future analysis of reaction and energy transfer pathways in complex systems.

\section{ACKNOWLEDGEMENTS}

This work was supported by the U.S. Department of Energy (DOE), Chemical Sciences, Geosciences and Biosciences Division, Office of Basic Energy Sciences under Grant No. DE-FG02-11ER16247 and the National Science Foundation (NSF) CAREER Award under Grant No. CHE-1057112. Additionally, T.F.M. acknowledges support from an Alfred P. Sloan Foundation Research Fellowship. Computational resources were provided by the National Energy Research Scientific Computing Center, which is supported by the Office of Science of the U.S. Department of Energy under Contract No. DE-AC02$05 \mathrm{CH} 11231$.

\section{APPENDIX I: QUAPI IMPLEMENTATION FOR PCET}

Here, we describe the implementation of the QUAPI method used in the current study. The total PCET Hamiltonian in Eq. (9) is written as a sum of system and bath contributions such that, $H=H_{s}+H_{B}$, where

$$
H_{\mathrm{s}}=\frac{p_{s}^{2}}{2 m_{s}}+\frac{p_{x}^{2}}{2 m_{x}}+V_{\mathrm{e}}(x, s)+V_{\mathrm{p}}(x)+V_{\mathrm{ps}}(x, s)
$$


and the Hamiltonian describing the bath modes and their coupling to the solvent coordinate is

$$
H_{\mathrm{B}}=\sum_{j=1}^{f} \frac{P_{j}^{2}}{2 M_{j}}+\sum_{j=1}^{f} \frac{1}{2} M_{j} \omega_{j}^{2}\left(Q_{j}-\frac{c_{j} s}{M_{j} \omega_{j}^{2}}\right)^{2} .
$$

In practice, we report the symmetrized form of the fluxflux correlation functions and note that the zeroth moment of these functions is identical to that of the standard correlation function defined in Eq. 4421. The symmetrized FCC function is given by

$$
C_{j m}(t)=\operatorname{Tr}\left[F_{j} e^{i H t_{\mathrm{c}}^{*} / \hbar} F_{m} e^{-i H t_{\mathrm{c}} / \hbar}\right],
$$

where the subset indices $j, m \in\{\mathrm{i}, \mathrm{r}, \mathrm{p}\}$, and $t_{\mathrm{c}}=t-i \beta \hbar / 2$. The complex-time propagators in Eq. 21) are discretized into $\mathcal{N}$ time slices of length $\Delta t_{\mathrm{c}}$, and the trace over bath modes is evaluated analytically to yield

$$
\begin{aligned}
C_{j m}(t)= & \int d \mathbf{s} \int d \mathbf{x} \sum_{\boldsymbol{\sigma}=1}^{2} \mathcal{I}(\mathbf{s}) K\left(\mathbf{s}, \mathbf{x}, \boldsymbol{\sigma} ; t_{\mathrm{c}}\right) \\
& \times\left\langle s_{1}, x_{1}, \sigma_{1}\left|F_{j}\right| s_{2 \mathcal{N}+2}, x_{2 \mathcal{N}+2}, \sigma_{2 \mathcal{N}+2}\right\rangle \\
& \times \quad\left\langle s_{\mathcal{N}+2}, x_{\mathcal{N}+2}, \sigma_{\mathcal{N}+2}\left|F_{m}\right| s_{\mathcal{N}+1}, x_{\mathcal{N}+1}, \sigma_{\mathcal{N}+1}\right\rangle
\end{aligned}
$$

where $\mathbf{s}=\left\{s_{1}, \ldots, s_{2 \mathcal{N}+2}\right\}, \quad \mathbf{x}=\left\{x_{1}, \ldots, x_{2 \mathcal{N}+2}\right\}$, and $\boldsymbol{\sigma}=\left\{\sigma_{1}, \ldots, \sigma_{2 \mathcal{N}+2}\right\}$. Numerical evaluation of the integrals in Eq. 22 is performed using two independent path-integral Monte Carlo simulations (MC1 and MC2), as we have previously described in detail 33 .

The term $K\left(\mathbf{s}, \mathbf{x}, \boldsymbol{\sigma} ; t_{\mathrm{c}}\right)$ in Eq. 22 is the path-integral representation for the complex-time propagators of the system Hamiltonian,

$$
\begin{array}{r}
K\left(\mathbf{s}, \mathbf{x}, \boldsymbol{\sigma} ; t_{\mathrm{c}}\right)=\prod_{k=2}^{\mathcal{N}+1}\left\langle s_{k}, x_{k}, \sigma_{k}\left|e^{-i H_{\mathrm{s}} \Delta t_{c}}\right| s_{k-1}, x_{k-1}, \sigma_{k-1}\right\rangle \\
\quad \times \prod_{k=\mathcal{N}+3}^{2 \mathcal{N}+2}\left\langle s_{k}, x_{k}, \sigma_{k}\left|e^{i H_{\mathrm{s}} \Delta t_{c}^{*}}\right| s_{k-1}, x_{k-1}, \sigma_{k-1}\right\rangle,(23)
\end{array}
$$

where

$$
\begin{gathered}
\left\langle s_{k}, x_{k}, \sigma_{k}\left|e^{-i H_{\mathrm{s}} \Delta t_{\mathrm{c}} / \hbar}\right| s_{k-1}, x_{k-1}, \sigma_{k-1}\right\rangle= \\
\sum_{m=1}^{M_{0}} \phi_{m}\left(s_{k}, x_{k}, \sigma_{k}\right) \phi_{m}^{*}\left(s_{k-1}, x_{k-1}, \sigma_{k-1}\right) e^{-i E_{m} \Delta t_{\mathrm{c}} / \hbar},
\end{gathered}
$$

$\phi_{m}$ and $E_{m}$ are the eigenstates and eigenenergies of $H_{\mathrm{s}}$, respectively, and $M_{0}$ is the number of eigenstates included in the expansion. The eigenstates and eigenvalues are obtained from a three-dimensional DVR grid calculation in terms of the solvent coordinate $\left\{-s^{*},-s^{*}+\Delta s, \ldots, s^{*}-\Delta s, s^{*}\right\}$, the proton coordinate $\left\{-x^{*},-x^{*}+\Delta x, \ldots, x^{*}-\Delta x, x^{*}\right\}$, and the two electronic states.

The discretized form of the non-local influence functional in Eq. 22 is

$$
\mathcal{I}(\mathbf{s})=\mathcal{I}_{0} \exp \left(-\sum_{k=1}^{2 \mathcal{N}+2} \sum_{k^{\prime}=1}^{k} B_{k k^{\prime}} s_{k} s_{k^{\prime}}\right)
$$

where $\mathcal{I}_{0}$ is the partition function of the uncoupled bath oscillators $27|34| 35]$. For the case of linear system-bath coupling, the diagonal matrix elements are given by 27

$$
\begin{aligned}
B_{k k} & =\sum_{j=1}^{f} \frac{c_{j}^{2}}{M_{j} \omega_{j}^{3} \sinh \left(\beta \omega_{j} / 2\right)} \sin \left(\frac{\omega_{j}\left(t_{k+1}-t_{k}\right)}{2}\right) \\
& \times \sin \left(\frac{\omega_{j}\left(t_{k+1}-t_{k}+i \beta\right)}{2}\right),
\end{aligned}
$$

and the off-diagonal matrix elements are given by

$$
\begin{aligned}
B_{k k^{\prime}} & =\sum_{j=1}^{f} \frac{c_{j}^{2}}{M_{j} \omega_{j}^{3} \sinh \left(\beta \omega_{j} / 2\right)} \sin \left(\frac{\omega_{j}\left(t_{k+1}-t_{k}\right)}{2}\right) \\
& \times \cos \left(\frac{\omega_{j}\left(t_{k+1}-t_{k^{\prime}+1}+t_{k}-t_{k^{\prime}}+i \beta\right)}{2}\right) \\
& \times \sin \left(\frac{\omega_{j}\left(t_{k^{\prime}+1}-t_{k^{\prime}}\right)}{2}\right)
\end{aligned}
$$

The complex times $t_{k}$ in Eqs. 26) and (27) are provided in Table II

TABLE III. Complex times $t_{k}$ used to calculate the $\left\{B_{k k^{\prime}}\right\}$.

\begin{tabular}{cc}
\hline \hline$k$ & $t_{k}$ \\
\hline 1 & 0 \\
$2, \ldots, \mathcal{N}+1$ & $(k-1 / 2) \Delta t_{\mathrm{c}}$ \\
$\mathcal{N}+2$ & $t-i \beta \hbar / 2$ \\
$\mathcal{N}+3, \ldots, 2 \mathcal{N}+2$ & $(2 \mathcal{N}+3 / 2-k) \Delta t_{\mathrm{c}}^{*}-i \beta \hbar$ \\
$2 \mathcal{N}+3$ & $-i \beta \hbar$ \\
\hline \hline
\end{tabular}

The PCET flux operators, which correspond to the subset projection operators defined in Eqs. (15)-(17), are

$$
\begin{aligned}
F_{\mathrm{p}} & =F_{\mathrm{x}}|2\rangle\langle 2|+h(x) F_{\mathrm{e}} \\
F_{\mathrm{r}} & =-F_{\mathrm{x}}|1\rangle\langle 1|-h(-x) F_{\mathrm{e}}, \\
F_{\mathrm{i}} & =F_{\mathrm{x}}(|1\rangle\langle 1|-| 2\rangle\langle 2|)+(h(-x)-h(x)) F_{\mathrm{e}},
\end{aligned}
$$

where $F_{\mathrm{x}}=\frac{i}{\hbar}[H, h(x)]$ is the flux operator for the proton coordinate and $F_{\mathrm{e}}=\frac{i}{\hbar}[H,|2\rangle\langle 2|]$ is the flux operator for the electronic diabatic states. The matrix elements appearing in Eq. (28) are evaluated using

$$
\begin{aligned}
& \left\langle s_{k}, x_{k}, \sigma_{k}\left|F_{\mathrm{x}}\right| 2\right\rangle\left\langle 2 \mid s_{k^{\prime}}, x_{k^{\prime}}, \sigma_{k^{\prime}}\right\rangle= \\
& \quad \frac{i \hbar}{2 m_{x} x_{\mathrm{FD}}} \delta_{\sigma_{k}, 2} \delta_{\sigma_{k^{\prime}}, 2} \delta\left(s_{k}-s_{k^{\prime}}\right) \\
& \quad \times\left[\delta\left(x_{k}\right) \delta\left(x_{k^{\prime}}-x_{\mathrm{FD}}\right)-\delta\left(x_{k}-x_{\mathrm{FD}}\right) \delta\left(x_{k^{\prime}}\right)\right]
\end{aligned}
$$

where $x_{\mathrm{FD}}=\Delta x$, and

$$
\begin{aligned}
\left\langle s_{k}, x_{k}, \sigma_{k}\right. & \left.\left|h(x) F_{\mathrm{e}}\right| s_{k^{\prime}}, x_{k^{\prime}}, \sigma_{k^{\prime}}\right\rangle= \\
& \frac{i}{\hbar} h\left(x_{k}\right) \delta\left(x_{k}-x_{k^{\prime}}\right) V_{12}\left(s_{k}\right) \delta\left(s_{k}-s_{k^{\prime}}\right) \\
& \times\left[\delta_{\sigma_{k}, 1} \delta_{\sigma_{k^{\prime}}, 2}-\delta_{\sigma_{k}, 2} \delta_{\sigma_{k^{\prime}}, 1}\right]
\end{aligned}
$$

respectively. Similar expressions are used for the terms in Eqs. 29 and 30.

All convergence parameters for the QUAPI calculations are provided in Table IV Convergence with respect 
to the number of path-integral beads is determined by comparing FAC functions with $\mathcal{N}=3-5$; the employed value of $\mathcal{N}=4$ is consistent with the number for beads required in previous QUAPI simulations for proton and electron transfer reactions $29 / 31 / 35$. Convergence with respect to the eigenfunction expansion is determined by comparing the trace over the complex-time propagator with $M_{0}=100-2000$; no significant numerical changes are found for cutoffs larger than those reported in Table IV.

TABLE IV. Convergence parameters for QUAPI.

\begin{tabular}{cccccccc}
\hline \hline Model & $M_{0}$ & MC1 $^{\mathrm{a}}$ & $\mathrm{MC}^{\mathrm{a}}$ & $s^{*}$ & $\Delta s$ & $x^{*}$ & $\Delta x$ \\
\hline I & 500 & $1 \times 10^{7}$ & $1 \times 10^{8}$ & 5 & 0.1 & 4 & 0.2 \\
II & 750 & $1 \times 10^{8}$ & $5 \times 10^{8}$ & 11 & 0.09 & 1 & 0.14 \\
III & 500 & $1 \times 10^{7}$ & $1 \times 10^{8}$ & 5 & 0.1 & 4 & 0.2 \\
\hline \hline
\end{tabular}

a Indicates the number of Metropolis acceptance/rejection steps.

For numerical comparison, the rate of the concerted PCET reaction in model I is calculated using both the QUAPI method and a golden-rule rate expression. The QUAPI rate is obtained using

$$
k_{\mathrm{QUAPI}}=\frac{k_{\mathrm{rp}}}{Q_{\mathrm{r}}}
$$

where $k_{\mathrm{rp}}$ is defined in Eq. (6) and $Q_{\mathrm{r}}$ is the partition function for reactant subset $\Omega_{r}$; this yields a rate $k_{\mathrm{Q}}=1.7(1) \times 10^{-6}$ a.u. The golden-rule approximation for vibronically nonadiabatic PCET is given by $\underline{36} \underline{40}$

$$
\begin{aligned}
k_{\mathrm{GR}}=\sum_{\mu} & P_{\mu} \sum_{\nu} \frac{V_{\mu \nu}^{2}}{\hbar}\left(\frac{\beta \pi}{\lambda}\right)^{1 / 2} \\
& \times \exp \left[\frac{-\beta\left(\Delta G^{0}+\lambda+\epsilon_{\nu}-\epsilon_{\mu}\right)^{2}}{4 \lambda}\right],
\end{aligned}
$$

where $\epsilon_{\mu}$ and $\epsilon_{\nu}$ are the energies of the proton vibrational states $\mu$ and $\nu$, respectively, and $P_{\mu}$ is the thermal probability corresponding vibrational state $\mu$. The PCET reaction in model I is ground-state dominated, electronically adiabatic and vibrationally nonadiabatic; construction of the diabats for model I thus yields a vibronic coupling of $V_{00}=3.75 \times 10^{-4}$, solvent reorganization energy $\lambda=1.34 \times 10^{-2}$ a.u., and driving force $\Delta G^{0}=0$. The golden-rule rate obtained for the concerted reaction in model $\mathrm{I}$ is $k_{\mathrm{GR}}=2.06 \times 10^{-6}$ a.u., which is in good agreement with the QUAPI rate stated above.
${ }^{1}$ P. G. Bolhuis, D. Chandler, C. Dellago and P. L. Geissler, Ann. Rev. Phys. Chem. 53, 291 (2002).

${ }^{2}$ G. Hummer, J. Chem. Phys. 120, 516 (2004).

${ }^{3}$ T. S. Van Erp and P. G. Bolhuis, J. Comp. Phys. 205, 157 (2005).

${ }^{4}$ T. F. Miller, III and C. Predescu, J. Chem. Phys. 126, 144102 (2007).

${ }^{5}$ T. F. Miller, III, E. Vanden-Eijnden, and D. Chandler, Proc. Natl. Acad. USA, 104, 14559 (2007).

${ }^{6}$ E. Weinan, W. Ren, and E. Vanden-Eijnden, J. Chem. Phys. 126, 164103 (2007).

${ }^{7}$ S. H. Northrup and J. T. Hynes, J. Chem. Phys. 73, 2700 (1980). ${ }^{8}$ A. Mitra and H. Rabitz, Phys. Rev. A 67, 033407 (2003).

${ }^{9}$ P. Huo and D. F. Coker, J. Chem. Phys. 133, 184108 (2010).

${ }^{10}$ J. Yuen-Zhou, J. J. Krich, M. Mohseni, and A. Aspuru-Guzik, Proc. Natl. Acad. Sci. USA 108, 17615 (2011).

${ }^{11}$ N. Boekelheide, R. Salomon-Ferrer and T. F. Miller, III, Proc. Natl. Acad. Sci. USA 108, 16159 (2011).

${ }^{12}$ R. I. Cukier and D. G. Nocera, Ann. Rev. Phys. Chem. 49, 337 (1998).

${ }^{13}$ S. Hammes-Schiffer, Acc. Chem. Res. 34, 273 (2001).

${ }^{14}$ J. M. Mayer, Ann. Rev. Phys. Chem. 55, 363 (2004).

${ }^{15}$ M. H. Huynh and T. J. Meyer, Chem. Rev. 107, 5004 (2007).

${ }^{16}$ V. R. I. Kaila and G. Hummer, J. Am. Chem. Soc. 133, 19040 (2011).

17 J. Bonin and M. Robert, Photochem. Photobiol. 87, 1190 (2011).

${ }^{18}$ A. V. Soudackov, A. Hazra, and S. Hammes-Schiffer, J. Chem. Phys. 135, 144115 (2011).

${ }^{19}$ W. H. Miller, J. Chem. Phys. 61, 1823 (1974).

${ }^{20}$ G. A. Voth, D. Chandler and W. H. Miller, J. Chem. Phys. 91, 7749 (1989).

${ }^{21}$ W. H. Miller, S. D. Schwarz, and J. W. Tromp, J. Chem. Phys. 79, 4889 (1983).

${ }^{22}$ T. C. Germann and W. H. Miller, J. Phys. Chem. A 101, 6358 (1997).

${ }^{23}$ N. Ananth and T. F. Miller, III, J. Chem. Phys., 133, 234103 (2010).

${ }^{24}$ A. O. Caldeira and A. J. Leggett, Ann. Phys. 149, 374 (1983).

${ }^{25}$ I. R. Craig and D. E. Manolopoulos, J. Chem. Phys. 122, 084016 (2005).

${ }^{26}$ J.-Y. Fang and S. Hammes-Schiffer, J. Chem. Phys. 106, 8442 (1997).

${ }^{27}$ N. Makri, Chem. Phys. Lett. 183, 435 (1992).

${ }^{28}$ D. E. Makarov and N. Makri, Phys. Rev. A 48, 3626 (1993).

${ }^{29} \mathrm{M}$. Topaler and N. Makri, Chem. Phys. Lett. 210, 285 (1993).

${ }^{30} \mathrm{M}$. Topaler and N. Makri, Chem. Phys. Lett. 210, 448 (1993).

${ }^{31}$ M. Topaler and N. Makri, J. Phys. Chem. 100, 4430 (1996).

${ }^{32}$ S. Shin and S.-I. Cho, Chem. Phys. 259, 27 (2000).

${ }^{33}$ A. R. Menzeleev, N. Ananth, and T. F. Miller, III, J. Chem. Phys. 135, 074106 (2011).

${ }^{34}$ R. P. Feynman and F. L. Vernon, Ann. Phys. 24, 118 (1963).

${ }^{35}$ E. Sim, G. Krilov, and B. J. Berne, J. Phys. Chem. A 105, 2824 (2001).

${ }^{36} \mathrm{~J}$. Ulstrup, Charge transfer processes in condensed media (Springer-Verlag, New York, 1979).

${ }^{37}$ C. Costentin, M. Robert and J.-M. Saveant, J. Am. Chem. Soc. 129, 9953 (2007).

${ }^{38}$ C. Venkataraman, A. V. Soudackov and S. Hammes-Schiffer, J. Phys. Chem. C 112, 12386 (2008).

${ }^{39}$ S. Hammes-Schiffer and A. A. Stuchebrukhov, Chem. Rev. 110, 6939 (2010).

${ }^{40}$ D. Borgis and J. T. Hynes, J. Phys. Chem. 100, 1118 (1996).

${ }^{41}$ D. T. Colbert and W. H. Miller, J. Chem. Phys. 96, 1982 (1992). 